\title{
Short-term step-to-step correlation in plantar pressure distributions during treadmill walking, and implications for footprint trail analysis
}

\author{
Todd C. Pataky ${ }^{1}$, Russel Savage ${ }^{2}$, Karl T. Bates ${ }^{2}$, William I. Sellers ${ }^{3}$, and Robin H. Crompton ${ }^{2}$ \\ ${ }^{1}$ Department of Bioengineering, Shinshu University, Japan \\ ${ }^{2}$ Department of Musculoskeletal Biology, Institute of Ageing and Chronic Disease, University of Liverpool, UK \\ ${ }^{3}$ Faculty of Life Sciences, University of Manchester
}

\begin{abstract}
The gait cycle is continuous, but for practical reasons one is often forced to analyze one or only a few adjacent cycles, for example in non-treadmill laboratory investigations and in fossilized footprint analysis. The nature of variability in long-term gait cycle dynamics has been wellinvestigated, but short-term variability, and specifically correlation, which are highly relevant to short gait bouts, have not. We presently tested for step-to-step autocorrelation in a total of 5243 plantar pressure (PP) distributions from ten subjects who walked at $1.1 \mathrm{~m} / \mathrm{s}$ on an instrumented treadmill. Following spatial foot alignment, data were analyzed both from three points of interest (POI): heel, central metatarsals, and hallux, and for the foot surface as a whole, in a mass-univariate manner. POI results revealed low average step-to-step autocorrelation coefficients $(\mathrm{r}=0.327 \pm 0.094$; mean \pm st. dev.). Formal statistical testing of the whole-foot $\mathrm{r}$ distributions reached significance over an average of only $0.42 \pm 0.52 \%$ of the foots surface, even for a highly conservative uncorrected threshold of $p<0.05$. The common assumption, that short gait bouts consist of independent cycles, is therefore not refuted by the present PP results.
\end{abstract}




\section{Introduction}

Fossilized footprints provide important information regarding the gait of extinct species, but often contain only a few consecutive steps. Non-treadmill laboratory investigations are similarly limited to measuring only a few consecutive gait cycles. In such investigations, it may not be appropriate to assume that neighboring footprints and gait cycles are independent.

Long-term structure in gait dynamics has been well documented, most prominently for interstride duration $[1-3]$. The analyses $[1-3]$ indicate that gait variability is regulated deterministically over hundreds of gait cycles, during both treadmill and overground walking [2], as evidenced by disruptions to long-term dynamics due to neurological impairment [3]. These studies also imply that short-term effects are considerably weaker than long-term effects [3], but do not reject the possibility of statistical significance in short-term correlations.

The purpose of this study was to determine the strength of short-term correlations in neighboring gait cycles during short gait bouts. Since short gait bouts are common to both clinical and footprint applications, we presently use a proxy for gait regularity that most closely links these two applications: plantar pressure (PP) distribution variability. Specifically, we test the null hypothesis that PP values at specific locations are uncorrelated across multiple steps. Rejection of this hypothesis would imply that neighboring footsteps/gait cycles should not be regarded as independent observations.

\section{Methods}

\section{$2.1 \quad$ Experiment}

Five females and five males participated in this experiment $(28.9 \pm 4.2$ years, $170.2 \pm 11.3 \mathrm{~cm}$, $72.5 \pm 14.1 \mathrm{~kg}$ ), providing informed consent according to the policies of the University of Liverpool (approval \#RETH000888). Subjects performed ten $30 \mathrm{~s}$ bouts of $1.1 \mathrm{~m} / \mathrm{s}$ walking on a Mercury treadmill (H/P/Cosmos, Germany) that was instrumented with an FDM-T pressure measurement device (Zebris Medical, Germany; $100 \mathrm{~Hz}$ ), yielding an average of 524.3 PP distributions per subject. 


\subsection{Data processing}

Raw PP data were linearly interpolated to moving-foot coordinate systems using treadmill belt displacement encoder data. Maximal PP distributions were then registered (i.e. optimally aligned; Fig.1a), first within-feet using a frequency-domain technique [4], and then between-feet (and withinsubjects) using a nonlinear technique [5] to minimize left-right morphological asymmetries.

The resulting discrete times series of PP distributions were then analyzed using two separate approaches: "point of interest" (POI) and "whole foot". The former assessed maximal Heel, central metatarsals (MT), and Hallux via manual POI digitizing followed by a search in a five-pixel radius for the local maxima. The latter assessed all pixels simultaneously, in a topological manner [6-8] as outlined below.

\subsection{Statistical analysis}

The mean within-foot PP distribution was made the datum to avoid left-right asymmetry effects. POI time-series (Fig. 1b) were then auto-regressed (Fig. 1c) using time lags k to represent short gait bouts $(0<\mathrm{k}<5)$. Statistical power for these autocorrelations was also assessed [9], assuming effect sizes equivalent to the observed slopes.

For "whole-foot" analysis the above was repeated for all pixels, yielding autocorrelation coefficient $(r)$ distributions (Fig. 1d), which were then converted to $t$ distributions (Fig. 1e) via the identity:

$$
t=r \sqrt{\frac{n-k-2}{1-r^{2}}}
$$

where $n$ is the number of steps, and where $t$ has Students $t$ distribution with degrees of freedom: $(n-k-2)$. Since $t$ is both parametric and pixel-specific it is referred to as a 'statistical parametric map' (SPM), and is denoted "SPM $\{t\}$ " [7]. Using $\alpha=0.05, \operatorname{SPM}\{t\}$ significance was assessed at each pixel in two separate ways: (i) an uncorrected threshold of $p<0.05$, and (ii) a Bonferronicorrected threshold of $p<0.0051$ across the ten subjects. The percentage of supra-threshold pixels 
(Fig. 1f) was used to summarize significance.

\section{Results}

\subsection{Experimental data}

The POI data exhibited weak autocorrelations, with a cross-bout maximum, lag-1 average of $r=0.327 \pm 0.094$, and cross-subject and cross-POI maximum of $r=0.542$ (Table 1a). Across subjects, statistical power values were $0.803 \pm 0.061,0.755 \pm 0.085$ and $0.809 \pm 0.086$ for the Heel, MT and Hallux, respectively.

Whole foot analysis yielded higher $r$ values, but none exceeded 0.863 (Table 1a). The autocorrelation distributions (Fig. 1d) revealed similarly low $r$ values across the foot surface (Fig. 2), irrespective of foot morphology, with slightly higher values in the low-pressure toe and midfoot regions. The only notable exception to this trend was the lag-1 autocorrelation of Subject 8, which exhibited a broad negative $r$ patch in the lateral foot; note that Fig. 2 depicts only absolute $r$ values.

Statistical inference via $\operatorname{SPM}\{t\}$ data (Fig. 1e and f) revealed an average supra-threshold area of only $0.42 \pm 0.52 \%$ of the total foot area (Table $1 \mathrm{~b}$ ), even for an uncorrected threshold of $\alpha=$ 0.05 across the ten subjects. Subject 8 notably exhibited average supra-threshold areas of only $2.4 \%$ and $1.3 \%$ for uncorrected and Bonferroni-corrected thresholds, respectively.

The aforementioned Subject 8 peculiarities could be explained by neither body mass nor body mass index (see Supplementary material). Additionally, since the supra-threshold foot area for this subject was well below $5 \%$ (Table $1 \mathrm{~b}$ ) - the amount that could be expected from purely random variation - these peculiarities were deemed to be outliers, and to lack practical significance.

\section{Discussion}

Maximal PP distributions during treadmill walking were presently found to exhibit weak shortterm autocorrelation, and we therefore fail to reject the null hypothesis that local PP values are uncorrelated in short gait bouts. For the present POIs, statistical power analysis found that the 
null hypothesis is likely true with an average probability of $78.9 \%$.

While these findings somewhat contrast observations of non-random long-term variance [1-3], the short- and long-term results are nevertheless compatible because the objects of study, and quite likely the modes of mediation, are quite different: the present short-term correlation results simply reflect step-to-step mechanical co-variation, whilst previous long-term results reflect neurally mediated nonlinear dynamics underlying motor organization [3]. In steady-state walking lowerthan-average pressures would be expected to follow higher-than-average pressures, and vice versa, only if local pressure were an explicit control variable. It seems more reasonable to expect that pressures are modulated in a long-term manner, possibly to avoid excessive cumulative loading.

The whole-foot approach was presently found to be more conservative than the POI approach (Table 1a) because the former identified true lattice-wide statistical optima. Thus the whole-foot approach appears to be more appropriate, not just for avoiding false positives [8], but also for avoiding false negatives.

It is presently unclear why relatively high $r$ values were observed in the low-pressure toe and midfoot regions (Fig. 2). It is possible that this is an artifact of the lower signal-to-noise ratios associated with low-pressure measurements.

One limitation of this study was that PP cannot map directly to footprint depth [10]. Partially mitigating this limitation are similarities between treadmill and over-ground walking [2]. Regardless, we believe that the present results are valuable because they provide the first explicit support for assumptions of short-term gait-cycle independence.

A second limitation was that correlation was not assessed between anatomical regions (e.g. heel and hallux). Such anatomical cross-correlation techniques are well-established in the statistics [6] and neuroimaging literatures [7], and similar parametric techniques have been validated elsewhere for both PP $[8,11]$ and general biomechanical continua [12]. Nevertheless, cross-correlation is left for future work because inter-region covariance is typically analyzed for neither PP nor footprint data.

In summary, the present results failed to provide evidence that local PP is linearly dependent on its values in up to four previous steps. This provides support for the common assumption of 
independence amongst neighboring gait cycles in short gait bouts, and also agrees with findings that several footsteps are needed to accurately estimate mean PP distributions [13].

\section{Acknowledgments}

Financial support for this work was provided by Royal Society grant \#IE111087 and Natural Environment Research Council grant \#NE/H004246/1.

\section{Conflict of Interest}

The authors report no conflict of interest. None of the authors has a financial or personal relationship with other people or organizations that could have inappropriately biased this work.

\section{Appendix A. Supplementary data}

Supplementary data are attached below.

\section{References}

[1] Hausdorff JM, Peng CK, Ladin Z, Wei JY, Goldberger AL. Is walking a random walk? Evidence for long-range correlations in stride interval of human gait. Journal of Applied Physiology 1995; 78(1): 349-58.

[2] Bollens B, Crevecoeur F, Nguyen V, Detrembleur C, Lejeune T. Does human gait exhibit comparable and reproducible long-range autocorrelations on level ground and on treadmill? Gait and Posture 2010; 32(3): $369-73$.

[3] Hausdorff JM, Mitchell SL, Firtion R, Peng CK, Cudkowicz ME, Wei JY, Goldberger AL. Altered fractal dynamics of gait: reduced stride-interval correlations with aging and Huntingtons disease. Journal of Applied Physiology 1997; 82(1): 262-9.

[4] Oliveira FPM, Pataky TC, Tavares JMRS. Registration of pedobarographic image data in frequency domain. Computer Methods in Biomechanics and Biomedical Engineering 2010;13(6):262-9.

[5] Pataky TC, Keijsers NLW, Goulermas JY, Crompton RH. Nonlinear spatial warping for between-subjects pedobarographic image registration. Gait and Posture 2009; 29: 477-82. 
[6] Adler RJ, Taylor JE. Random fields and geometry. New York: Springer; 2007.

[7] Friston KJ, Ashburner JT, Kiebel SJ, Nichols TE, Penny WD, editors. Statistical parametric mapping: the analysis of functional brain images. London: Elsevier; 2007.

[8] Pataky TC, Caravaggi P, Savage R, Parker D, Goulermas J, Sellers WI, Crompton RH. New insights into the plantar pressure correlates of walking speed using pedobarographic statistical parametric mapping (pSPM). Journal of Biomechanics 2008; 41(9): 1987-94.

[9] Dupont WD, Plummer WD. Power and sample size calculations for studies involving linear regression. Controlled Clinical Trials 1998; 19(6): 589-601.

[10] D'Août K, Meert L, Van Gheluwe B, De Clercq D, Aerts P. Experimentally generated footprints in sand: analysis and consequences for the interpretation of fossil and forensic footprints. American Journal of Physical Anthropology 2010; 141(4): 515-25.

[11] PatakyTC. Assessingthesignificanceofpedobarographicsignalsusingrandom field theory. Journal of Biomechanics $2008 ; 41(11):$ 2465-73.

[12] Pataky TC. Generalized $n$-dimensional biomechanical field analysis using statistical parametric mapping. Journal of Biomechanics 2010; 43(10): 1976-82.

[13] Arts MLJ, BusSA.Twelve steps per foot are recommended for valid and reliable in-shoe plantar pressure data in neuropathic diabetic patients wearing custom made footwear. Clinical Biomechanics 2011; 26(8): 880-4. 
(a)
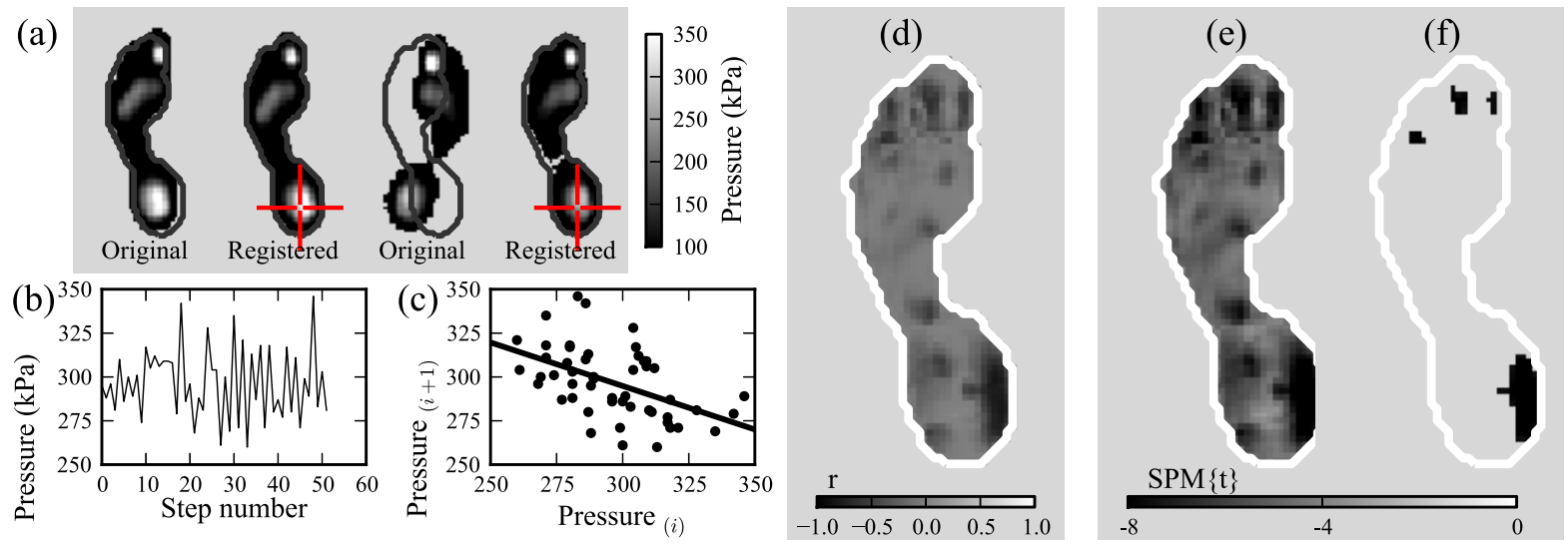

Figure 1: Methods overview, single subject (Subject 8). (a) Original left and right foot images were registered to the within-subject mean distribution; cross-hairs indicate the heel point-of-interest (POI). (b) Heel POI values for each step in a single treadmill bout. (c) Lag-1 autocorrelation of data in (b), with regression coefficient $r=-0.499$. (d) $r$ distribution. (e) Statistical parametric map of $t$ values $(\operatorname{SPM}\{t\})$, which map directly from $r$ values. (f) Inference image depicting which parts of the foot reached significance. 


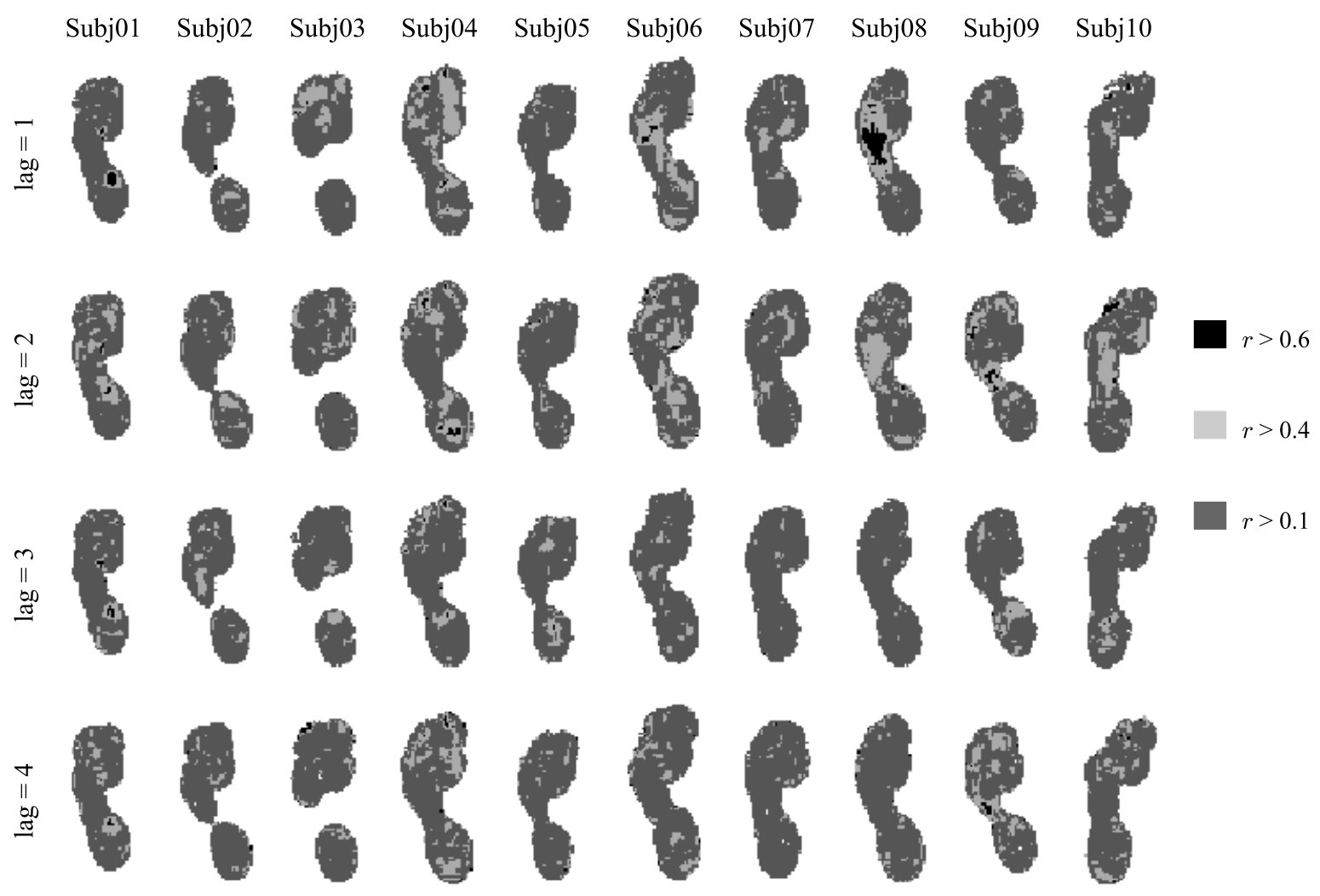

Figure 2: Autocorrelation coefficient $(r)$ distributions, depicting maximum $r$ values across ten trials. 
Table 1. Autocorrelation analysis summary. (a) Maximum autocorrelation coefficients ( $r)($ lag $=1$ step) across the ten trials, for three points of interest (POI): heel, central metatarsal heads (MT) and hallux. The maximum absolute $r$ value across the entire foot is also included. (b) Percentage of foot area exceeding the threshold for $r$ significance (see Fig.1d-f); results for both uncorrected and Bonferroni-corrected error rates of of $\alpha=0.05$ and of $\alpha=0.0051$, respectively, are presented.

\begin{tabular}{|c|c|c|c|c|c|c|c|c|}
\hline & \multicolumn{4}{|c|}{$\begin{array}{c}\text { (a) } \\
\text { Maximal autocorrelation coefficients }(r)\end{array}$} & \multicolumn{4}{|c|}{$\begin{array}{c}\text { (b) } \\
\text { Supra-threshold foot area }(\%)\end{array}$} \\
\hline & \multicolumn{3}{|c|}{ POI } & \multirow{2}{*}{$\begin{array}{l}\text { Whole } \\
\text { foot }\end{array}$} & \multicolumn{2}{|c|}{$\alpha=0.05$} & \multicolumn{2}{|c|}{$\alpha=0.0051$} \\
\hline & Heel & MT & Hallux & & $\operatorname{lag}=1$ & $\operatorname{lag}=2$ & $\operatorname{lag}=1$ & $\operatorname{lag}=2$ \\
\hline Subj01 & 0.304 & 0.246 & 0.181 & 0.707 & 0.5 & 0.6 & 0.4 & 0.2 \\
\hline Subj02 & 0.332 & 0.338 & 0.191 & 0.842 & 0.1 & 0.1 & 0.0 & 0.0 \\
\hline Subj03 & 0.363 & 0.450 & 0.344 & 0.669 & 0.5 & 0.1 & 0.1 & 0.0 \\
\hline Subj04 & 0.512 & 0.370 & 0.491 & 0.773 & 0.4 & 0.7 & 0.2 & 0.2 \\
\hline Subj05 & 0.275 & 0.429 & 0.286 & 0.658 & 0.0 & 0.1 & 0.0 & 0.1 \\
\hline Subj06 & 0.302 & 0.448 & 0.286 & 0.665 & 0.5 & 0.3 & 0.1 & 0.1 \\
\hline Subj07 & 0.238 & 0.305 & 0.398 & 0.567 & 0.2 & 0.1 & 0.0 & 0.0 \\
\hline Subj08 & 0.286 & 0.542 & 0.228 & 0.710 & 2.4 & 0.3 & 1.3 & 0.0 \\
\hline Subj09 & 0.242 & 0.271 & 0.256 & 0.863 & 0.0 & 1.0 & 0.0 & 0.5 \\
\hline Subj10 & 0.367 & 0.199 & 0.329 & 0.791 & 0.1 & 0.3 & 0.1 & 0.2 \\
\hline
\end{tabular}




\section{Supplementary Material}

The purpose of this supplementary is to address the issue of Subject 8's apparently peculiar result (Fig.2, main manuscript).

Subject 8 had a mass of $79 \mathrm{~kg}$, and body masses ranged from $59 \mathrm{~kg}$ to $95 \mathrm{~kg}$ in the present sample, so body mass itself was not peculiar to this subject. We were also unable to observe (albeit qualitatively) any systematic effect of body mass on average PP distributions (Fig.S1a).

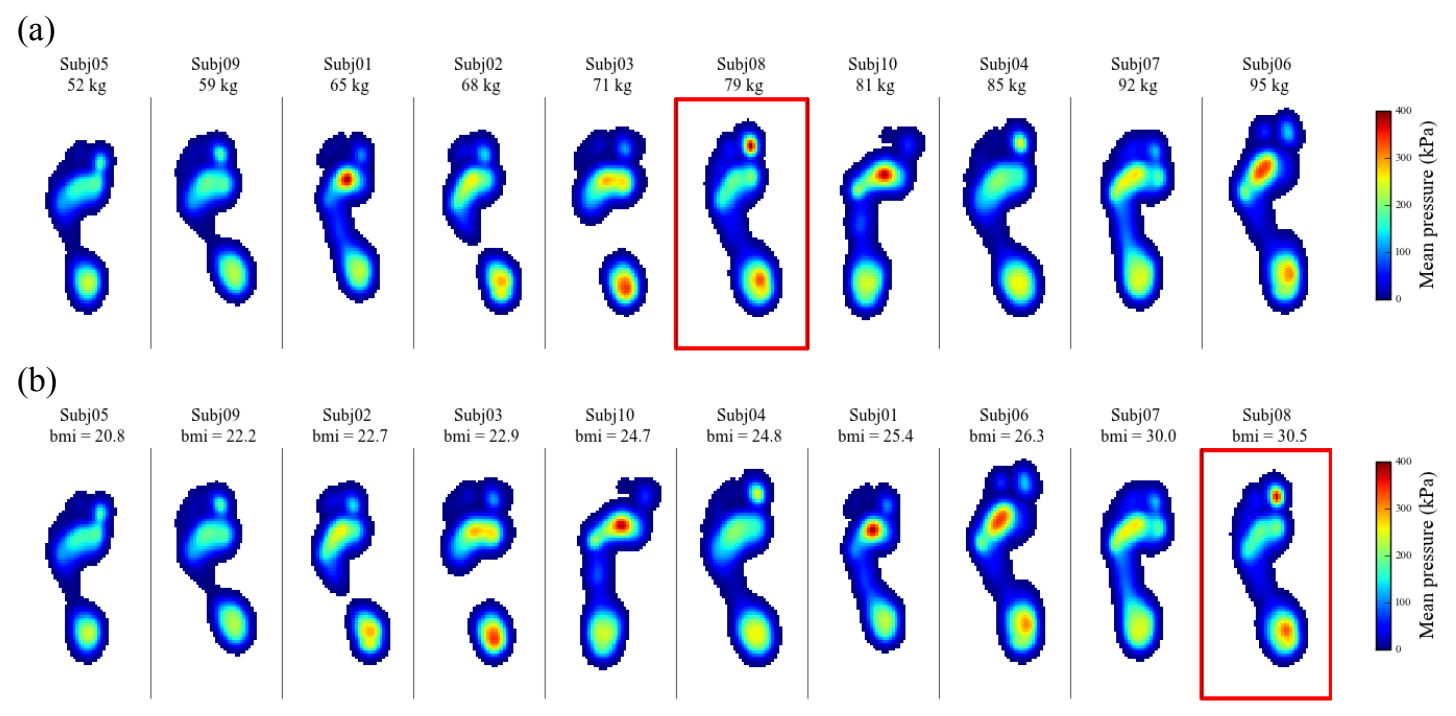

Figure S1. Average plantar pressure distribution for each of the ten subjects: (a) in order of increasing body mass, and (b) in order of increasing body mass index (BMI). Subject 8's data are highlighted.

Subject 8 had the highest body mass index (BMI) in the current sample. Subject 8 and Subject 7, with BMIs of 30.5 and 30.0, respectively, exceeded the standard cutoff for obesity (BMI $>30)$. Two other subjects were "overweight" $(25<\mathrm{BMI}<30)$, and the remaining subjects were "Normal". Nevertheless, as above there did not appear to be a systematic effect of BMI on PP distribution (Fig.S1b).

To further probe BMI issue we extracted pressure data from the point of maximum autocorrleation in Subject 8's data, which was found to lie in the posterior aspect of the lateral forefoot (Fig.2, main manuscript). For subsequent reference we shall refer to this point as "r_max(8)". To assess this point in a between-subject manner we manually digitized homologous points in the other nine subjects.

Results indicate, firstly, that Subject 8 had the highest r_max(8) pressure (Fig.S2). 
However the pressure magnitude at this point was lower than than at the other three POIs (Heel, MT and Hallux) in this subject, and was also higher than many other recorded pressures. The variability data at $\mathrm{r} \_\max (8)$ also indicate nothing peculiar about Subject 8 (Fig.S2, lower panels).
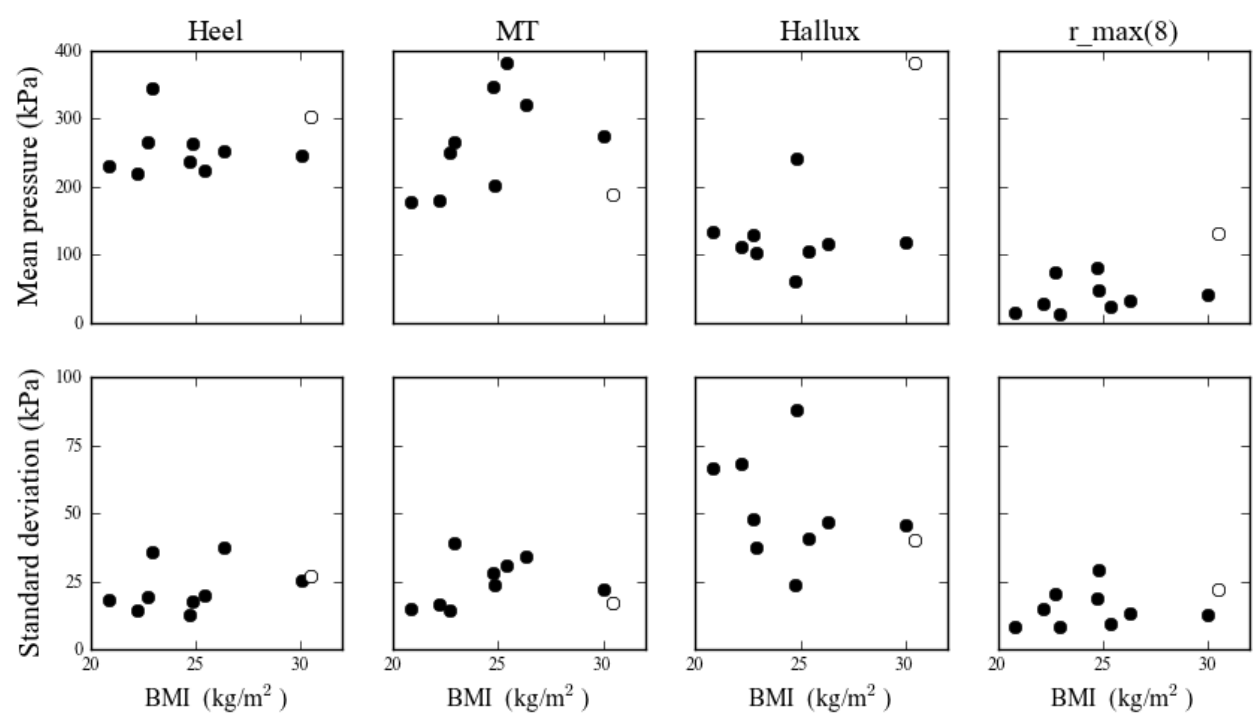

Figure S2. Point of interest (POI) pressure values for each of the ten subjects, means and standard deviations, in order of increasing BMI. Open circles depict Subject 8's data. The POI "r_max(8)" indicates the point of maximum autocorrelation in Subject 8 (lateral posterior forefoot; Fig.2). Points homologous to r_max $(8)$ were digitized manually in the other nine subjects.

In summary, if there is indeed a biomechanical explanation for the peculiar Subject 8 autocorrelation result in Fig.2 (main manuscript), then this this explanation does not appear to be a straightforward function of gross subject descriptors. We are therefore unable to derive a testable hypothesis, even in a post hoc sense, that would directly address the cause of this peculiar result.

Furthermore, if the presently observed autocorrelation coefficient distributions were governed by a completely random process, then we could expect that alpha $\%$ of the area of the total foot area would reach the critical statistical threshold simply by chance. We presently chose alpha $=0.05$, but observed that far less than $5 \%$ of the foot surface reached this threshold (Table 1b, main manuscript). Most importantly, even for Subject 8 considerably less than $5 \%$ of the foot area reached the uncorrected alpha $=0.05$ threshold, indicating that, even in this apparently peculiar subject, the autocorrelations were weaker than one would predict by chance. We therefore believe that it is justifiable to regard this apparently anomalous result as a chance occurrence, as simply a classical outlier. 